\title{
Universal Lefschetz fibrations and Lefschetz cobordisms
}

\author{
DANIELE ZUDDAS
}

\begin{abstract}
We construct universal Lefschetz fibrations, defined in analogy with classical universal bundles. We also introduce the cobordism groups of Lefschetz fibrations, and we see how these groups are quotients of the singular bordism groups via the universal Lefschetz fibrations.
\end{abstract}

$55 \mathrm{R} 55 ; 57 \mathrm{R} 90,57 \mathrm{~N} 13$

\section{Introduction}

Topological Lefschetz fibrations over surfaces have been given considerable attention in the last decade, because of their applications to symplectic and contact topology; see for example Akbulut and Ozbagci [1], Donaldson [8] Gompf and Stipsicz [17] Loi and Piergallini [20]. This led to several generalizations, including achiral Lefschetz fibrations and their relations with branched coverings and braided surfaces (Apostolakis, Piergallini and Zuddas [3], Fuller [13] and Zuddas [24]), broken Lefschetz fibrations (Baykur [4; 5] and Gay and Kirby [15]), and Morse 2-functions (Gay and Kirby [14; 16]). In Di Scala, Kasuya and Zuddas [7], Matsumoto's torus fibration on $S^{4}$ [21] (see also Gompf and Stipsicz [17, Example 8.4.7]) was used to construct an almost complex structure on $\mathbb{R}^{4}$ containing holomorphic tori.

We are going to further generalize Lefschetz fibrations by allowing the base manifold to have arbitrary dimension. The critical image of a Lefschetz fibration is a codimension2 submanifold of the target manifold, and the monodromy is a homomorphism to the mapping class group of the fiber. Actually, to understand the larger amount of information that a generalized Lefschetz fibration carries with respect to a standard one (that is, over a surface), we need several types of monodromies, each one capturing some aspects, but not others.

Universal Lefschetz fibrations were introduced in Zuddas [25] in analogy with universal bundles, under the additional assumption that the base surface had nonempty boundary. The purpose of the present paper is twofold: to relax this restriction by allowing the base surface to be closed, and to start building a (co)bordism theory for Lefschetz fibrations along the lines of classical bordism theory. Our main results include a 
characterization of universal Lefschetz fibrations in dimension two (Theorem 2.1) and three (Theorem 2.3), an explicit construction of these fibrations, and an application to Lefschetz cobordism groups (that are defined in Section 4), proving that these groups are quotients of certain singular bordism groups in dimension two and three (Proposition 4.6 and Corollary 4.7). We will give some computations, and further developments, in a forthcoming paper.

Throughout this paper all manifolds and maps are assumed to be smooth. We consider only oriented compact manifolds and (local) diffeomorphisms that preserve the orientations, unless stated otherwise.

Acknowledgements The work presented in this paper has been partially carried out at the Max Planck Institute for Mathematics in Bonn, Germany, during the 4-manifolds semester (January-June 2013). I would like to thank the institute for its support and hospitality. Thanks also to Mark Grant (Newcastle University) for a useful answer on MathOverflow.

\section{Definitions, preliminaries and notations}

By the standard definition, a Lefschetz fibration is, roughly speaking, a smooth map over a surface with only nondegenerate (possibly achiral) complex singularities. In order to state our results we propose the following generalization.

For $f: V \rightarrow M$, we denote by $\tilde{A}_{f} \subset V$ the critical set of $f$, and by $A_{f}=f\left(\tilde{A}_{f}\right)$ the critical image of $f$.

Definition 1.1 Let $M$ and $V$ be manifolds of dimensions $m+2$ and $m+2 k$ respectively, with $m \geq 0$ and $k \geq 2$. A Lefschetz fibration $f: V \rightarrow M$ is a map such that:

(1) Near any critical point $\tilde{a} \in \tilde{A}_{f}, f$ is locally equivalent to the map

$$
f_{0}: \mathbb{R}_{+}^{m} \times \mathbb{C}^{k} \rightarrow \mathbb{R}_{+}^{m} \times \mathbb{C}, \quad f_{0}\left(x, z_{1}, \ldots, z_{k}\right)=\left(x, z_{1}^{2}+\cdots+z_{k}^{2}\right),
$$

where $x \in \mathbb{R}_{+}^{m}=\left\{\left(x_{1}, \ldots, x_{m}\right) \in \mathbb{R}^{m} \mid x_{m} \geq 0\right\}$ and $\left(z_{1}, \ldots, z_{k}\right) \in \mathbb{C}^{k}$.

(2) $f_{\mid}: \tilde{A}_{f} \rightarrow M$ is an embedding.

(3) $f_{\mid}: V-f^{-1}\left(A_{f}\right) \rightarrow M-A_{f}$ is a locally trivial bundle whose fiber is a manifold $F$ (the regular fiber of $f$ ).

Note that when $A_{f}=\varnothing, f$ is an honest bundle. 
Definition 1.2 We call $f_{\mid}: V-f^{-1}\left(A_{f}\right) \rightarrow M-A_{f}$ the regular bundle associated with $f$.

We see below that there is also a singular bundle associated with $f$. The following proposition is a simple consequence of the definition.

Proposition 1.3 Let $f: V \rightarrow M$ be a Lefschetz fibration.

(1) $\tilde{A}_{f}$ is a proper submanifold of $V$ of dimension $m$.

(2) $A_{f}$ is a proper submanifold of $M$ of codimension two.

(3) $f_{\mid}: \tilde{A}_{f} \rightarrow A_{f}$ is a diffeomorphism.

(4) The regular fiber $F \subset V$ is a submanifold of dimension $2 k-2$.

For $m=0, f$ is an ordinary (possibly achiral) Lefschetz fibration. So, a generalized Lefschetz fibration looks locally as an ordinary one times an identity map. Throughout the paper we assume $k=2$. This implies that $F$ is a surface.

In general, $A_{f}$ can be nonorientable. However, if $A_{f}$ is orientable, by fixing an orientation on it (hence on $\tilde{A}_{f}$ via $f_{\mid}: \tilde{A}_{f} \rightarrow A_{f}$ ) we can define the positive and the negative critical points and values: $\widetilde{a} \in \tilde{A}_{f}$ is a positive critical point of $f$ if the local coordinates considered in the definition can be chosen to be compatible with the orientations of $V, M$, and $A_{f}$ (that corresponds to $\mathbb{R}^{m} \times\{0\} \subset \mathbb{R}^{m} \times \mathbb{C}$ in the local chart). Otherwise, $\tilde{a}$ is said to be a negative critical point. Accordingly, $a=f(\widetilde{a})$ is said to be a positive or negative critical value. This positivity or negativity is locally invariant, hence the connected components of $A_{f}$ inherit it.

Two Lefschetz fibrations $f_{1}: V_{1} \rightarrow M_{1}$ and $f_{2}: V_{2} \rightarrow M_{2}$ are said to be equivalent if there are orientation-preserving diffeomorphisms $\phi: V_{1} \rightarrow V_{2}$ and $\psi: M_{1} \rightarrow M_{2}$ such that $\psi \circ f_{1}=f_{2} \circ \phi$. This implies that $\psi\left(A_{f_{1}}\right)=A_{f_{2}}$ and that $\phi\left(\widetilde{A}_{f_{1}}\right)=\tilde{A}_{f_{2}}$. If $A_{f_{1}}$ and $A_{f_{2}}$ are oriented, we assume also that $\psi_{1}: A_{f_{1}} \rightarrow A_{f_{2}}$ is orientation-preserving. If $f_{1}$ and $f_{2}$ are equivalent, we make use of the notation $f_{1} \cong f_{2}$.

Let $f: V \rightarrow M$ be a Lefschetz fibration whose regular fiber is the oriented surface $F=F_{g, b}$ of genus $g$ with $b$ boundary components, and let $N$ be a $n$-manifold.

Definition 1.4 A map $q: N \rightarrow M$ is said to be $f$-regular if $q$ and $q_{\mid \partial N}$ are transverse to $f$.

If $q: N \rightarrow M$ is $f$-regular then $\tilde{V}=\{(x, v) \in N \times V \mid q(x)=f(v)\}$ is a $(n+2)-$ manifold and the map $\widetilde{f}: \widetilde{V} \rightarrow N$ defined by $\tilde{f}(x, v)=x$ is a Lefschetz fibration. The map $\tilde{q}: \tilde{V} \rightarrow V$ defined by $\widetilde{q}(x, v)=v$ sends each fiber of $\tilde{f}$ diffeomorphically onto a fiber of $f$, hence the regular fiber of $\tilde{f}$ is still $F$. Moreover, we have $A_{\tilde{f}}=q^{-1}\left(A_{f}\right)$. 
Definition 1.5 We say that $\tilde{f}$ is the pullback of $f$ by $q$. We denote it by $\tilde{f}=q^{*}(f)$.

Let $\mathcal{L}(F)$ be some class of Lefschetz fibrations with fiber $F$.

Definition 1.6 We say that a Lefschetz fibration $u$ : $U \rightarrow M$ with fiber $F$ is $\mathcal{L}(F)$ universal (or universal with respect to $\mathcal{L}(F)$ ) if:

(1) For any $f: V \rightarrow N$ that belongs to $\mathcal{L}(F)$, there exists a $u$-regular map $q: N \rightarrow$ $M$ such that $q^{*}(u) \cong f$.

(2) Any such pullback for an arbitrary $q: N \rightarrow M$ belongs to $\mathcal{L}(F)$ up to equivalence, where $N$ is the base of a Lefschetz fibration of $\mathcal{L}(F)$.

In other words, $u$ is $\mathcal{L}(F)$-universal if and only if the class $\mathcal{L}(F)$ coincides with the class of pullbacks of $u$ obtained by those maps $q: N \rightarrow M$ such that $N$ is the base of a Lefschetz fibration that belongs to $\mathcal{L}(F)$.

Monodromies Now we consider connected Lefschetz fibrations. The nonconnected ones can be easily handled by restricting to connected components.

Let $\mathcal{M}_{g, b}$ be the mapping class group of $F_{g, b}$, namely the group of self-diffeomorphisms of $F_{g, b}$ which keep $\partial F_{g, b}$ fixed pointwise, up to isotopy through such diffeomorphisms. Let also $\widehat{\mathcal{M}}_{g, b}$ be the general mapping class group of $F_{g, b}$, whose elements are the isotopy classes of orientation-preserving self-diffeomorphisms of $F_{g, b}$ (without assumptions on the boundary).

The regular bundle associated with $f: V \rightarrow M$ has a monodromy homomorphism $\widehat{\omega}_{f}: \pi_{1}\left(M-A_{f}\right) \rightarrow \widehat{\mathcal{M}}_{g, b}$.

Definition 1.7 We call $\widehat{\omega}_{f}$ the bundle monodromy of $f$.

For a codimension-2 submanifold $A \subset M$, let $N(A)$ be a compact tubular neighborhood of $A$ in $M$, endowed with its disk bundle structure $B^{2} \hookrightarrow N(A) \rightarrow A$. Take a base point $* \in M-N(A)$, and let $N(*) \subset M-N(A)$ be a small ball around $*$. We join $N(*)$ with each component of $N(A)$ by a narrow 1-handle, and let $\bar{N}(A)$ be the result. By construction, the manifold $\bar{N}(A)$ is uniquely determined, up to diffeomorphisms, by the normal bundle of $A$ in $M$, although its embedding in $M$ in general is not unique. If $A$ is connected, we have $\bar{N}(A) \cong N(A)$.

We denote by $\mu_{1}(M, A)$ the subgroup of $\pi_{1}(M-A)$ generated by the meridians of $A$ in $M$. Note that $\mu_{1}(M, A)$ is the kernel of the homomorphism induced by the inclusion $i_{*}: \pi_{1}(M-A) \rightarrow \pi_{1}(M)$, so it is a normal subgroup.

Let $f: V \rightarrow M$ be a Lefschetz fibration, and let $\bar{f}: \bar{V} \rightarrow \bar{N}\left(A_{f}\right)$ be the restriction of $f$ over $\bar{N}\left(A_{f}\right)$. 
Taking a fiber of $N\left(A_{f}\right) \rightarrow A_{f}$, that is a transverse 2-disk $B^{2}$, the restriction of $f$ over it is a Lefschetz fibration $f^{\prime}: V^{\prime} \rightarrow B^{2}$ with only one critical point. So its monodromy is a Dehn twist [17] about a curve $c \subset F$, which is said to be a vanishing cycle. Thus, the singular fiber is homeomorphic to $F / c$. The vanishing cycles that correspond to different components of $A_{f}$ might be topologically different as embedded curves in $F$. However, the local model of $f$ near a critical point implies that the restriction of $f$ over a component of $A_{f}$ is a locally trivial bundle over that component with fiber $F / c$ (the total space is not a topological manifold).

Definition 1.8 We call $f_{\mid}: f^{-1}\left(A_{f}\right) \rightarrow A_{f}$ the singular bundle associated with $f$.

Note that the singular fiber $F / c$ is homeomorphic to a (possibly disconnected) surface $F_{c}$, with two points $p_{1}$ and $p_{2}$ identified. The surface $F_{c}$ is obtained by surgering $F$ along $c$. Moreover, any self-homeomorphism of $F / c$ lifts to a unique homeomorphism of $\left(F_{c},\left\{p_{1}, p_{2}\right\}\right)$. If $c$ is nonseparating, then $F_{c} \cong F_{g-1, b}$ is connected, so in this case the monodromy of the singular bundle is a homomorphism $\omega_{f}^{\bowtie:} \pi_{1}\left(A_{f}\right) \rightarrow \widehat{\mathcal{M}}_{g-1, b, 2}$, where $\widehat{\mathcal{M}}_{g, b, n}$ denotes the general mapping class group of $F_{g, b}$ with $n$ marked points (mapping classes are allowed to permute the marked points and the boundary components). In general, we have to consider the general mapping class group of a surface with two marked points and with at most two components (each one containing a marked point).

Definition 1.9 We call $\omega_{f}^{\bowtie}$ the singular monodromy of $f$. If $A_{f}$ is not connected, we define $\omega_{f}^{\bowtie}$ to be the set of singular monodromies of the components of $A_{f}$.

Remark If the vanishing cycles are all nonseparating and $g \geq 2$, the singular bundle is determined by the singular monodromy.

We already know that the monodromy of a meridian of $A_{f}$ in $\bar{N}\left(A_{f}\right)$ is a Dehn twist. Then there is a canonical homomorphism $\omega_{f}: \mu_{1}\left(\bar{N}\left(A_{f}\right), A_{f}\right) \rightarrow \mathcal{M}_{g, b}$ that sends a meridian to the corresponding Dehn twist.

Definition 1.10 We call $\omega_{f}$ the Lefschetz monodromy of $f$.

We say that $f$ is an allowable Lefschetz fibration if the monodromy of an arbitrary meridian of $A_{f}$ is a Dehn twist about a curve $c \subset F$ that is homologically essential in $F$. For the sake of simplicity, we assume that the Lefschetz fibrations we consider are allowable unless stated otherwise. However, most of the results of this paper hold also in the nonallowable case, by suitable modifications.

Consider the canonical homomorphism $\beta: \mathcal{M}_{g, b} \rightarrow \widehat{\mathcal{M}}_{g, b}$ that sends a mapping class $[\phi] \in \mathcal{M}_{g, b}$ to the mapping class $[\phi] \in \widehat{\mathcal{M}}_{g, b}$. 
The Lefschetz and the bundle monodromies are related by the commutative diagram

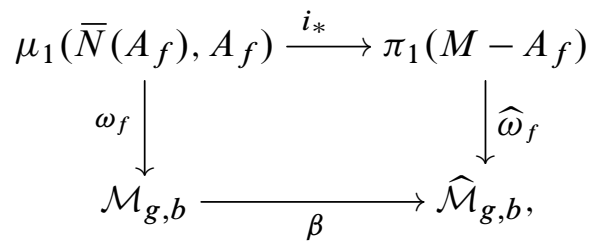

where $i_{*}$ is induced by the inclusion $i: \bar{N}\left(A_{f}\right)-A_{f} \hookrightarrow M-A_{f}$.

There is also a compatibility condition between the bundle monodromy and the singular monodromy. Roughly speaking, the monodromy of a loop contained in $N\left(A_{f}\right)$ must preserve the vanishing cycle associated to this component.

Let $\Pi_{1}(F)=\pi_{1}(\operatorname{Diff}(F)$, id $)$. We say that $F$ is exceptional if $\Pi_{1}(F) \neq 0$. It is known that $F_{g, b}$ is exceptional if and only if $(g, b) \in\{(0,0),(0,1),(0,2),(1,0)\}$; see for example [18]. However, an allowable Lefschetz fibration with the disk or the sphere for a fiber is necessarily an honest bundle, and for this reason we assume that the fiber is not the sphere or the disk. So, the only exceptional fibers we admit are the torus and the annulus. Moreover, for any $(g, b) \neq(0,0), \pi_{i}\left(\operatorname{Diff}\left(F_{g, b}\right)\right.$,id $)=0$ for all $i>1$, $\Pi_{1}\left(T^{2}\right) \cong \mathbb{Z}^{2}$, and $\Pi_{1}\left(S^{1} \times I\right) \cong \mathbb{Z}$; see $[9 ; 10 ; 18]$.

To state our results, we need a further invariant of Lefschetz fibrations. Consider an element $[\alpha] \in \pi_{2}\left(M-A_{f}\right), \alpha: S^{2} \rightarrow M-A_{f}$, and let $\tilde{f}=\alpha^{*}(f): \tilde{V} \rightarrow S^{2}$. It follows that $\tilde{f}$ is a locally trivial $F$-bundle. Decompose $S^{2}=D_{1} \cup_{\partial} D_{2}$ as the union of two disks $D_{1}$ and $D_{2}$, and trivialize $\tilde{f}$ over $D_{i}$, that is $\tilde{f}^{-1}\left(D_{i}\right) \cong D_{i} \times F$. The two trivializations differ by an element $\tau \in \Pi_{1}(F)$ along $\partial D_{1}=\partial D_{2} \cong S^{1}$. This defines a homomorphism $\omega_{f}^{s}: \pi_{2}\left(M-A_{f}\right) \rightarrow \Pi_{1}(F)$, such that $\omega_{f}^{s}([\alpha])=\tau$. This homomorphism is exactly the one that fits into the homotopy exact sequence of the associated $\operatorname{Diff}(F)$-bundle over $M-A_{f}$.

Definition 1.11 We call $\omega_{f}^{s}$ the structure monodromy of $f$.

Now, consider the pullback $\tilde{f}=q^{*}(f)$, with $f: V \rightarrow M$ and $q: N \rightarrow M$ being base point preserving. Let $q_{*}: \pi_{i}(N) \rightarrow \pi_{i}(M)$, and let $q_{\mid *}: \pi_{i}\left(N-A_{\tilde{f}}\right) \rightarrow \pi_{i}\left(M-A_{f}\right)$ and $q_{\mid *}: \pi_{1}\left(A_{\tilde{f}}\right) \rightarrow \pi_{1}\left(A_{f}\right)$ be the homomorphisms induced by the restrictions $q_{\mid}: N-$ $A_{\tilde{f}} \rightarrow M-A_{f}$ and $q_{\mid}: A_{\tilde{f}} \rightarrow A_{f}$ (we consider the collection of these homomorphisms when $A_{\tilde{f}}$ is not connected).

The following proposition is simple and its proof is left to the reader. 
Proposition 1.12 Suppose that $q\left(\bar{N}\left(A_{\tilde{f}}\right)\right) \subset \bar{N}\left(A_{f}\right)$. We have

$$
\begin{gathered}
q_{*}\left(\mu_{1}\left(\bar{N}\left(A_{\tilde{f}}\right), A_{\tilde{f}}\right)\right) \subset \mu_{1}\left(\bar{N}\left(A_{f}\right), A_{f}\right), \\
\omega_{\tilde{f}}=\omega_{f} \circ q_{\mid *}, \quad \widehat{\omega}_{\tilde{f}}=\widehat{\omega}_{f} \circ q_{\mid *}, \quad \omega_{\tilde{f}}^{s}=\omega_{f}^{s} \circ q_{\mid *} .
\end{gathered}
$$

Moreover, the singular bundle of $\tilde{f}$ is the pullback of the singular bundle of $f$ by $q_{\mid A_{\tilde{f}}}$, hence $\omega_{\widetilde{f}}^{\bowtie}=\omega_{f}^{\bowtie} \circ q_{\mid *}$.

The twisting operation Consider a Lefschetz fibration $f: V \rightarrow M$ with exceptional fiber $F$. Let $\psi \in \Pi_{1}(F)$. We are going to construct a new Lefschetz fibration $f_{\psi}: V_{\psi} \rightarrow M$. Consider an oriented 2-disk $D \subset M-A_{f}$, and take a tubular neighborhood $C \times B^{m-1}$ of $C=\partial D$, with $m=\operatorname{dim} M$. Fix the (isotopically unique) trivialization of $f$ over $C \times B^{m-1}$ that extends over $D$. This determines a fiberwise embedded copy of $C \times B^{m-1} \times F$ in $V$, that is the preimage of $C \times B^{m-1}$. Now, twist $f$ over $C$ by means of $\psi$. To do this, remove $\operatorname{Int}\left(C \times B^{m-1} \times F\right)$ from $V$, and glue it back differently by composing the original attaching diffeomorphism to the right with $\Psi: C \times B^{m-1} \times F \rightarrow C \times B^{m-1} \times F$, defined by $\Psi(x, y, z)=\left(x, y, \psi_{x}(z)\right)$, where (up to some identifications) $\psi: C \rightarrow \operatorname{Diff}(F), \psi: x \mapsto \psi_{x}$, satisfies $\psi_{x_{0}}=\mathrm{id}_{F}$ for some $x_{0} \in C$.

What we get is a new Lefschetz fibration $f_{\psi}: V_{\psi} \rightarrow M$. We call $f_{\psi}$ the twisting of $f$ by $\psi$. The twisting operation was considered by Moishezon in [22] as one of the main tools needed to classify positive genus- 1 Lefschetz fibrations over the 2 -sphere. See also, for example, [19] for similar use in the context of achiral fibrations.

Remark By results of Moishezon [22, Part II], the twisting of $f$ is equivalent to $f$ if $\omega_{f}$ is surjective.

Theorem 1.13 Let $L=(M, A, F, \omega, \widehat{\omega}, \zeta)$ be the data of:

- A codimension-2 submanifold $A \subset M$.

- A nonexceptional connected surface $F$.

- Two homomorphisms $\omega: \mu_{1}(\bar{N}(A), A) \rightarrow \mathcal{M}(F)$ and $\widehat{\omega}: \pi_{1}(M-A) \rightarrow \widehat{\mathcal{M}}(F)$ that fit into the commutative diagram (1-1).

- A bundle $\zeta$ over $A$ with fiber $F / c$, where $c \subset F$ a simple curve that depends on the component of $A$, such that $\zeta$ is compatible with $\omega$ and $\widehat{\omega}$ in the above sense.

Then there exists a Lefschetz fibration $f_{L}: V_{L} \rightarrow M_{L}$ with fiber $F$, uniquely determined by $L$ up to equivalence, such that $A_{f_{L}}=A, \omega_{f_{L}}=\omega, \widehat{\omega} f_{L}=\widehat{\omega}$, and having singular bundle equivalent to $\zeta$. 
Moreover, for another such data $L^{\prime}=\left(M^{\prime}, A^{\prime}, F^{\prime}, \omega^{\prime}, \widehat{\omega}^{\prime}, \zeta^{\prime}\right)$ we have $f_{L} \cong f_{L^{\prime}}$ if and only if there are diffeomorphisms $\psi:(M, A, *) \rightarrow\left(M^{\prime}, A^{\prime}, *^{\prime}\right)$ sending $\bar{N}(A)$ onto $\bar{N}\left(A^{\prime}\right)$, and $h: F \rightarrow F^{\prime}$ such that:

(1) $\zeta \cong \zeta^{\prime}$ by a bundle equivalence that covers $\psi_{\mid A}: A \rightarrow A^{\prime}$.

(2) $\omega^{\prime} \circ \psi_{1 *}=h_{*} \circ \omega$ and $\widehat{\omega}^{\prime} \circ \psi_{2 *}=h_{*} \circ \widehat{\omega}$, where the $\psi_{i *}$ are the isomorphisms induced by $\psi_{1}=\psi_{\mid}: \bar{N}(A)-A \rightarrow \bar{N}\left(A^{\prime}\right)-A^{\prime}$ and $\psi_{2}=\psi: M-A \rightarrow M^{\prime}-A^{\prime}$ respectively on the fundamental group, and $h_{*}$ is the canonical isomorphism induced by $h$ between the relevant mapping class groups $h_{*}: \mathcal{M}(F) \cong \mathcal{M}\left(F^{\prime}\right)$ or $h_{*}: \widehat{\mathcal{M}}(F) \cong \widehat{\mathcal{M}}\left(F^{\prime}\right)$.

If $F$ is exceptional, the same holds up to twistings.

Actually, this is a consequence of known general facts in fiber bundles theory, so we give only a sketch of the proof.

Sketch of proof By the classical theory of fiber bundles, $\widehat{\omega}$ determines uniquely an $F$-bundle over $M-\operatorname{Int} N(A)$; see for example [12, Chapter 5]. On the other hand, $\omega$ and $\zeta$ determine a Lefschetz fibration over $\bar{N}(A)$. Glue these fibrations by a suitable fibered diffeomorphism. This proves the existence. For the uniqueness, notice that any such fibered diffeomorphism extends to the interior of $N(A)$. Indeed, this is well-known in dimension two, and working on the tubular neighborhood $N(A)$ thought as a disk bundle $B^{2} \hookrightarrow N(A) \rightarrow A$, one can adapt the two-dimensional case in a fiberwise fashion. Of course, the only ambiguity occurs when $F$ is exceptional, and this can be handled by a suitable twisting.

Note that the twisting action of $\Pi_{1}(F)$ is transitive on the set of possible structure monodromies for a fixed $(M, A, F, \omega, \widehat{\omega})$. However, the structure monodromy cannot be used to resolve the ambiguity of the twisting action, as it can be easily seen by considering genus- 1 Lefschetz fibrations over a closed surface.

Hurwitz systems and the monodromy sequence By a Hurwitz system for a codimension-2 submanifold $A \subset M$ we mean a sequence $\left(\xi_{1}, \ldots, \xi_{n} ; \eta_{1}, \ldots, \eta_{k}\right)$, where $\left\{\xi_{1}, \ldots, \xi_{n}\right\}$ are meridians of $A$ that normally generate $\mu_{1}(\bar{N}(A), A)$, and $\left\{\eta_{1}, \ldots, \eta_{k}\right\}$ are generators for $\pi_{1}(M-A)$ which are nontrivial in $\pi_{1}(M)$.

Once we fix a Hurwitz system, the Lefschetz and the bundle monodromies of $f$ can be represented by a sequence of Dehn twists and mapping classes $\left(\delta_{1}, \ldots, \delta_{n} ; \gamma_{1}, \ldots, \gamma_{k}\right)$ that we call the monodromy sequence of $f$. The elements of the monodromy sequence are given by $\delta_{i}=\omega_{f}\left(\xi_{i}\right) \in \mathcal{M}_{g, b}$, and $\gamma_{i}=\widehat{\omega}_{f}\left(\eta_{i}\right) \in \widehat{\mathcal{M}}_{g, b}$.

The Dehn twist $\delta_{i}$ is determined by a curve $c_{i} \subset F$, and by its sign. The curves $\left(c_{1}, \ldots, c_{n}\right)$ are the vanishing cycles of $f$ with respect to the given Hurwitz system. 


\section{The characterization theorems}

We denote by $\mathcal{C}_{g, b}$ the finite set of equivalence classes of homologically essential curves in $F=F_{g, b}$ up to orientation-preserving diffeomorphisms of $F$. Note that $\# \mathcal{C}_{g, b}=1$ if $b \in\{0,1\}$.

Theorem 2.1 A Lefschetz fibration $u: U \rightarrow M$ with regular fiber $F$ is universal with respect to the class of Lefschetz fibrations over a surface and with fiber $F$, if the following three conditions hold:

(1) $\widehat{\omega}_{u}$ is an isomorphism.

(2) $\omega_{u}$ and $\omega_{u}^{s}$ are surjective.

(3) Any class of $\mathcal{C}_{g, b}$ can be represented by a vanishing cycle of $u$.

On the other hand, as a partial converse, $u$ being universal implies (2), (3), and the surjectivity of $\widehat{\omega}_{u}$.

In particular, for $g \geq 2$ and $b \in\{0,1\}, u$ is universal if $\widehat{\omega}_{u}$ is an isomorphism and $\omega_{u}$ is surjective.

It follows that there exist universal Lefschetz fibrations for any fiber.

The surjectivity of $\omega_{u}^{s}$ means that any locally trivial $F_{g, b}$-bundle over $S^{2}$ is the pullback of $u$ by a map $S^{2} \rightarrow M-A_{u}$.

Remark If $u$ is universal we cannot conclude that $\widehat{\omega}_{u}$ is an isomorphism. The reason is that any Lefschetz fibration can be embedded in a larger Lefschetz fibration by preserving the universality. For example we can add a 1 -handle $H^{1}$ to the base (if it has boundary), along with a fiberwise attachment of $H^{1} \times F$ to the total manifold. So, we can add nontrivial elements to ker $\widehat{\omega}_{u}$.

This theorem generalizes the following proposition, which was proved in [25].

Proposition 2.2 A Lefschetz fibration $u: U \rightarrow S$ over a surface with regular fiber $F$ is universal with respect to bounded base surfaces if and only if the following two conditions are satisfied:

(1) $\omega_{u}$ and $\widehat{\omega}_{u}$ are surjective.

(2) Any class of $\mathcal{C}_{g, b}$ can be represented by a vanishing cycle of $u$. 
Proof of Theorem 2.1 Suppose that $u: U \rightarrow M$ satisfies the conditions of the statement and let $f: V \rightarrow S$ be a Lefschetz fibration with regular fiber $F_{g, b}$ over a surface $S$.

If $S$ is closed let $S^{\prime} \subset S$ be the complement of a disk in $S$, with $A_{f} \subset$ Int $S^{\prime}$, and let $f^{\prime}: V^{\prime}=f^{-1}\left(S^{\prime}\right) \rightarrow S^{\prime}$ be the restriction of $f$ over $S^{\prime}$. Otherwise, if $S$ has boundary, put $S^{\prime}=S$ and $f^{\prime}=f$.

We claim that there is a connected surface $G \subset M$ transverse to $A_{u}$, such that $G \cap \bar{N}\left(A_{u}\right)$ is connected, the meridians of $A_{u}$ that are contained in $G \cap \bar{N}\left(A_{u}\right)$ normally generate $\mu_{1}\left(\bar{N}\left(A_{u}\right), A_{u}\right)$, and $G$ is $\pi_{1}$-surjective in $M$.

We start the construction of $G$ by considering a 2-disk $G_{0} \subset \bar{N}\left(A_{u}\right)-A_{u}$ centered at *. Then we attach 2-dimensional bands $G_{1}, \ldots, G_{n} \subset \bar{N}\left(A_{u}\right)$, each one representing a meridian of $A_{u}$ so that they normally generate $\mu_{1}\left(\bar{N}\left(A_{u}\right), A_{u}\right)$. The band $G_{i}$ is attached to $G_{0}$ along an arc for each $i \geq 1$. Then we attach suitable 2-dimensional orientable 1-handles to $G_{0}$ (chosen to be disjoint from $A_{u}$ ) which realize a finite set of generators for $\pi_{1}(M)$. The resulting surface satisfies the conditions of the claim.

Now consider the Lefschetz fibration $u^{\prime}=u_{\mid}: U^{\prime} \rightarrow G$ which is the restriction of $u$ over $G$, with $U^{\prime}=u^{-1}(G) \subset U$. It turns out that $u^{\prime}$ satisfies the conditions (1) and (2) of Proposition 2.2, hence $u^{\prime}$ is universal for Lefschetz fibrations over bounded surfaces. Then $f^{\prime} \cong\left(q^{\prime}\right)^{*}\left(u^{\prime}\right)=\left(q^{\prime}\right)^{*}(u)$ for a $u$-regular map $q^{\prime}: S^{\prime} \rightarrow G \subset M$.

The loop $\beta=q^{\prime}\left(\partial S^{\prime}\right)$, homotoped to represent an element of $\pi_{1}\left(M-A_{u}\right)$, satisfies $\widehat{\omega}_{u}(\beta)=1$. Therefore, $\beta$ is trivial in $\pi_{1}\left(M-A_{u}\right)$ because $\widehat{\omega}_{u}$ is an isomorphism. So, the map $q^{\prime}$ extends to a $u$-regular map $q: S \rightarrow M$ such that $q\left(S-S^{\prime}\right) \subset M-A_{u}$.

Now, if $F$ is not exceptional, we immediately conclude that $f=q^{*}(u)$, proving that $u$ is universal.

Otherwise, if $F$ is exceptional, $q^{*}(u)$ differs from $f$ by a twisting determined by an element $\psi \in \Pi_{1}(F)$. Since $\omega_{u}^{s}$ is surjective, there is a map $q^{\prime \prime}: S^{2} \rightarrow M-A_{u}$ such that $\omega_{u}^{s}\left(\left[q^{\prime \prime}\right]\right)=\psi$.

Up to a small homotopy relative to $q^{-1}\left(A_{u}\right)$, we can assume that there is a small disk $D \subset S$ such that $q_{\mid D}: D \rightarrow M-A_{u}$ is an embedding. Similarly, we can assume that there is a small disk $D^{\prime} \subset S^{2}$ such that $q_{\mid D^{\prime}}^{\prime \prime}$ is an embedding.

We can form the connected sum $q^{\prime \prime \prime}=q \# q^{\prime \prime}: S \# S^{2} \cong S \rightarrow M$ by identifying $\partial D$ with $\partial D^{\prime}$, and by connecting their images by a tube contained in $M-A_{u}$. It follows that $\left(q^{\prime \prime \prime}\right)^{*}(u) \cong f$. 
We now prove the partial converse. Let $u$ : $U \rightarrow M$ be universal with fiber $F_{g, b}$. By letting $S$ to be a suitable surface with boundary, it can be easily constructed a Lefschetz fibration $f: V \rightarrow S$ such that $\widehat{\omega}_{f}$ and $\omega_{f}$ are surjective, and such that any element of $\mathcal{C}_{g, b}$ can be represented by a vanishing cycle (meaning that there are sufficiently many critical points of $f$ ). Since $f=q^{*}(u)$ for some $u$-regular map $q: S \rightarrow M$, it immediately follows that $\widehat{\omega}_{u}$ and $\omega_{u}$ are surjective, and condition (3) of the statement.

Regarding the surjectivity of $\omega_{u}^{s}$, this immediately follows by representing arbitrary $F_{g, b}$-bundles over $S^{2}$ by a pullback of $u$ (regarding a surface bundle as a Lefschetz fibration without critical points).

Finally, the last sentence follows by the discussion in next section.

In case of Lefschetz fibrations over 3-manifolds we have the following result.

Theorem 2.3 Let $u: U \rightarrow M$ be a Lefschetz fibration with fiber $F$ which satisfies the following conditions:

(1) $\widehat{\omega}_{u}$ and $\omega_{u}^{s}$ are isomorphisms (so $\pi_{2}\left(M-A_{u}\right)=0$ for $F$ not exceptional).

(2) $\omega_{u}$ and $\omega_{u}^{\bowtie}$ are surjective.

(3) Any class of $\mathcal{C}_{g, b}$ can be represented by a vanishing cycle of $u$.

(4) $A_{u}$ is connected.

Then $u$ is universal for Lefschetz fibrations over 3-manifolds with fiber $F$.

Proof Let $f: V \rightarrow Y$ be a Lefschetz fibration, with $Y$ a connected 3-manifold. We want to show that $f$ is a pullback of $u$. The critical image $L=A_{f}$ is a curve in $Y$, that is a disjoint union of circles and arcs.

Claim If $Y$ is closed there is a handle decomposition of the form

$$
Y=H^{0} \cup n_{1} H^{1} \cup n_{2} H^{2} \cup H^{3}
$$

such that:

(1) $H^{0} \cap L$ is a possibly empty set of trivial arcs.

(2) $H^{1} \cap L$ is either empty or the core of $H^{1}$ for any 1-handle.

(3) $H^{i} \cap L=\varnothing$ for any higher-index handle. 
Sketch of a proof of the claim Start from an arbitrary handle decomposition, with only one 0 -handle and one 3-handle. Observe that, up to isotopy, we can assume that $L$ is disjoint from the 2 - and the 3 -handles, and that its intersection with any 1 -handle is either empty or a number of parallel copies of its core. It is now straightforward to add new 1-handles and complementary 2-handles to normalize the intersections with the 1-handles. By adding canceling pairs of 1- and 2-handles again we can normalize also the intersection with the 0 -handle, and this proves the claim.

Now proceed with the proof of the theorem. First, by taking the double, we can assume that $Y$ is closed. Consider a handle decomposition of $Y$ as that of the claim.

Over $H^{0}, f$ is a product $f_{0} \times$ id: $V_{0} \times I \rightarrow B^{2} \times I \cong H^{0}$, with $f_{0}: V_{0} \rightarrow B^{2}$ a Lefschetz fibration. It follows that $f_{0}$ is a pullback of $u$, because $u$ is universal for Lefschetz fibrations over a surface by Theorem 2.1. So, there is a $u$-regular map $q: H^{0} \rightarrow M$ such that $q^{*}(u)=f_{\mid H^{0}}$.

Next, we extend this map $q$ handle by handle, and after each step we continue to denote by $q$ also the extension. If $H^{1}$ does not intersect $L$, the monodromy of a loop that passes through it geometrically once can be easily realized by a map to $M-A_{u}$ that extends $q$ because $\widehat{\omega}_{u}$ is surjective, and this map trivially extends over the 1 -handle. If $H^{1}$ intersects $L$, we can find an arc in $A_{u}$ between the two endpoints $q\left(S^{0} \times\{0\}\right)$, where $H^{1}=B^{1} \times B^{2} \supset S^{0} \times\{0\}$. This arc can be suitably chosen to realize the singular monodromy of $f$ along the core of $H^{1}$, by using the fact that $A_{u}$ is connected and $\omega_{u}^{\bowtie}$ is surjective. This means that $q$ can be extended over the core of $H^{1}$, hence to $H^{1}$.

Extending $q$ to the $2-$ handles is possible because $\widehat{\omega}_{u}$ is an isomorphism. If $F$ is exceptional, we might also need to modify the map $q$ on $H^{2}$ in order to adjust the twisting, by an argument similar to that in the proof of Theorem 2.1.

Finally, extending $q$ to the 3 -handle $H^{3}$ is also possible because over the attaching sphere $\Sigma$ of $H^{3}, f$ is a trivial bundle. So, $\left[q_{\mid \Sigma}\right] \in \operatorname{ker} \omega_{u}^{s}=0$, and this implies that $q_{\mid \Sigma}: \Sigma \rightarrow M-A_{u}$ is homotopic to a constant in $M-A_{u}$. Therefore, $q$ can be extended over $H^{3}$.

We get $q: Y \rightarrow M$ which is $u$-regular, such that $f=q^{*}(u)$.

\section{Construction of universal Lefschetz fibrations}

Now we give explicit constructions of universal Lefschetz fibrations. First, we handle the case of Lefschetz fibrations over a surface, and for the sake of simplicity we assume $b \in\{0,1\}$, although a similar construction can be made in general. Thereafter, we extend this construction to dimension three. 
Dimension 2 Consider a finite presentation of $\mathcal{M}_{g, b}=\left\langle\delta_{1}, \ldots, \delta_{k} \mid r_{1}, \ldots, r_{l}\right\rangle$ with generators $\delta_{1}, \ldots, \delta_{k}$ and relators $r_{1}, \ldots, r_{l}$. We assume that each $\delta_{i}$ corresponds to a positive or negative Dehn twist about a nonseparating curve in $F_{g, b}$. Note that in this case $\# \mathcal{C}_{g, b}=1$.

If $b=1$, a presentation of $\widehat{\mathcal{M}}_{g, 1}$ can be obtained from that of $\mathcal{M}_{g, 1}$ by adding as a further relator the Dehn twist $r_{0}$ about a boundary parallel curve, expressed in terms of the generators $\delta_{i}$, that is, $\widehat{\mathcal{M}}_{g, 1}=\left\langle\delta_{1}, \ldots, \delta_{k} \mid r_{0}, r_{1} \ldots, r_{l}\right\rangle$, where $r_{0}$ should be substituted by a product of the form $r_{0}=\delta_{i_{1}}^{\epsilon_{1}} \cdots \delta_{i_{p}}^{\epsilon_{p}}$, with $i_{j} \in\{1, \ldots, k\}$ and $\epsilon_{j} \in\{-1,1\}$. Otherwise, if $b=0$, we have $\widehat{\mathcal{M}}_{g, 0}=\mathcal{M}_{g, 0}$.

Now, consider a Lefschetz fibration $v: V \rightarrow B^{2}$ with regular fiber $F_{g, b}$ and $k$ critical values, having $\left(\delta_{1}, \ldots, \delta_{k}\right)$ as the monodromy sequence with respect to some Hurwitz system. By abusing notation, we also denote by $\left(\delta_{1}, \ldots, \delta_{k}\right)$ the elements of the Hurwitz system. That is, we consider $\pi_{1}\left(B^{2}-A_{v}\right)=\left\langle\delta_{1}, \ldots, \delta_{k}\right\rangle$.

By Proposition 2.2, $v$ is universal for Lefschetz fibrations with regular fiber $F_{g, b}$ over bounded surfaces.

Put $v^{\prime}=\mathrm{id} \times v: B^{2} \times V \rightarrow B^{2} \times B^{2} \cong B^{4}$. Clearly $v^{\prime}$ is a Lefschetz fibration with regular fiber $F_{g, b}$, and it is universal with respect to bounded base surfaces. Moreover $A_{v^{\prime}}=B^{2} \times A_{v}$ is a set of mutually parallel trivial disks in $B^{4}$.

Each relator $r_{i}$ is a word in the generators $\delta_{i}$, so it can be represented by an embedded loop $\lambda_{i}$ in $S^{3}-\partial A_{v^{\prime}}$. Moreover, up to homotopy, we can assume that the loops $\lambda_{i}$ are pairwise disjoint.

Note that $\omega_{v^{\prime}}$ and $\widehat{\omega}_{v^{\prime}}$ are surjective. In order to kill the kernel of $\widehat{\omega}_{v^{\prime}}$ we add a 2-handle $H_{i}^{2}$ to $B^{4}$ along $\lambda_{i}$ with an arbitrary framing (for example with framing 0 ), for all $i$. Let $M_{2}$ be the resulting 4-manifold.

Let $L_{i}=H_{i}^{2} \cap S^{3}$ be the attaching region of $H_{i}^{2}$. Now, attach the trivial bundle $H_{i}^{2} \times F_{g, b}$ to $B^{2} \times V$ by a fiberwise identification $L_{i} \times F_{g, b} \cong\left(v^{\prime}\right)^{-1}\left(L_{i}\right)$. This is possible because $\lambda_{i}$ has trivial bundle monodromy. Let $U_{2}$ be the resulting 6-manifold. We get a new Lefschetz fibration $u_{2}: U_{2} \rightarrow M_{2}$ defined by $v^{\prime}$ in $B^{2} \times V \subset U_{2}$, and by the projection onto the first factor in $H_{i}^{2} \times F_{g, b}$ for all $i$.

If $F_{g, b}$ is not exceptional, by Theorem 2.1 we immediately conclude that $u_{2}$ is universal.

If $F$ is exceptional, in our situation we have $F=T^{2}$, and so $\Pi_{1}\left(T^{2}\right) \cong \mathbb{Z}^{2}$ [18]. The two generators of this group correspond to two oriented torus bundles $q_{1}$ and $q_{2}$ over $S^{2}$. Making the fiber sum of $u_{2}$ with $\mathrm{id}_{B^{2}} \times q_{1}$ and $\operatorname{id}_{B^{2}} \times q_{2}$ produces a Lefschetz fibration, which we still denote by $u_{2}: U_{2} \rightarrow M_{2}$, that satisfies all the conditions of Theorem 2.1, hence a universal one. 
Dimension 3 Start with the Lefschetz fibration $v^{\prime}$ of the above construction. First, we make the critical image connected. Since the Dehn twists $\delta_{i}$ are conjugate to each other, we can add a suitable oriented band between the $i^{\text {th }}$ and the $(i+1)^{\text {st }}$ disks of $A_{v^{\prime}}$, so that the monodromy extends over this band. After adding these bands for all $i \leq k-1$, we get a Lefschetz fibration $v^{\prime \prime}: V^{\prime \prime} \rightarrow B^{4}$. Note that $A_{v^{\prime \prime}}$ is a ribbon disk in $B^{4}$.

At this point, we want to make the singular monodromy surjective. To do this, we modify also the base manifold as follows. Consider a finite set of generators for the general mapping class group of $F_{g, b} / c \cong F_{g-1, b}$ with two marked points, where $c$ is a vanishing cycle of $v^{\prime \prime}$, and take two points $a_{1}, a_{2} \in \partial A_{v^{\prime \prime}}$, chosen to be very close to each other.

Let $g: Y \rightarrow B^{2}$ be a Lefschetz fibration with fiber $F$, having 0 as the only critical value, with monodromy given by that of a meridian of $\partial A_{v^{\prime \prime}}$ in $S^{3}$ that bounds a disk in $S^{3}$ with center at the point $a_{1}$.

Add a 1 -handle $H^{1}=B^{1} \times B^{1} \times B^{2} \cong B^{1} \times B^{3}$ to $B^{4}$, with attaching sphere $\left\{a_{1}, a_{2}\right\}$. Then $v^{\prime \prime}$ extends over $H^{1}$ by the product $\operatorname{id}_{B^{1}} \times \operatorname{id}_{B^{1}} \times g: B^{1} \times B^{1} \times Y \rightarrow H^{1}$. Actually, we attach $H^{1}$ trivially around $a_{1}$ and by realizing one generator of the mapping class group of $F / c$ around $a_{2}$. This is straightforward by taking into account the local product structure of $v^{\prime \prime}$ near $a_{1}$ and $a_{2}$. Proceed in a similar way to realize any generator by attaching further 1 -handles.

We end with a Lefschetz fibration $v^{\prime \prime \prime}$ such that $A_{v^{\prime \prime \prime}}$ is connected (and of genus 0), the singular monodromy is surjective, and the Lefschetz and bundle monodromies are surjective. So, after adding suitable 2-handles, we make the bundle monodromy an isomorphism.

If $F=T^{2}$ we have to make $\omega_{v^{\prime \prime \prime}}^{s}$ surjective, and this can be done by fiber sum with two torus bundles over the sphere, multiplied by the identity map, in analogy with the construction in dimension two.

We obtain a Lefschetz fibration $u^{\prime}: U^{\prime} \rightarrow M^{\prime}$ over a 4-manifold $M^{\prime}$, which is universal for Lefschetz fibrations over surfaces.

As the last step, we have to kill the kernel of $\omega_{u^{\prime}}^{s}$. To do this, simply take a finite set of generators for $\operatorname{ker}\left(\omega_{u^{\prime}}^{s}\right)$ and let $\alpha: S^{2} \rightarrow M^{\prime}-A_{u^{\prime}}$ be such a generator.

Consider the product $u^{\prime \prime}=u^{\prime} \times \operatorname{id}_{B^{2}}: U^{\prime} \times B^{2} \rightarrow M^{\prime} \times B^{2}$. In the 5-manifold $M^{\prime} \times S^{1} \subset \partial\left(M^{\prime} \times B^{2}\right)$ the map $\alpha$ can be perturbed to an embedding, so it can be represented by an embedded sphere $\Sigma \subset M^{\prime} \times S^{1}-A_{u^{\prime \prime}}$. Add a 3 -handle $H^{3}$ along this sphere, and extend $u^{\prime \prime}$ over $H^{3}$ by a trivial $F$-bundle. This is possible because $\Sigma$ 
is in the kernel of $\omega_{u^{\prime \prime}}^{s}$. Continue in this way to kill all the generators of the kernel. We end with a Lefschetz fibration $u_{3}: U_{3} \rightarrow M_{3}$ over a 6-manifold $M_{3}$ which is universal for 3-dimensional bases.

\section{Lefschetz cobordism}

For a Lefschetz fibration $f: V \rightarrow M$ we denote by $-f:(-V) \rightarrow(-M)$ the same Lefschetz fibration between the same manifolds with reversed orientation. Note that $f$ and $-f$ have the same oriented fiber. Let $f_{1}: V_{1} \rightarrow M_{1}$ and $f_{2}: V_{2} \rightarrow M_{2}$ be Lefschetz fibrations with fiber $F_{g}=F_{g, 0}$ such that $\operatorname{dim} M_{1}=\operatorname{dim} M_{2}=m$, and with $M_{i}$ and $V_{i}$ closed.

Definition 4.1 We say that $f_{1}$ and $f_{2}$ are cobordant if there exists a Lefschetz fibration $f: W \rightarrow Y$ with the same fiber $F_{g}$ such that $\partial W=V_{1} \sqcup\left(-V_{2}\right), \partial Y=M_{1} \sqcup\left(-M_{2}\right)$, and $f_{\mid \partial W}=f_{1} \sqcup\left(-f_{2}\right): V_{1} \sqcup\left(-V_{2}\right) \rightarrow M_{1} \sqcup\left(-M_{2}\right)$. In particular, if $f_{2}=\varnothing$, we say that $f_{1}$ is cobordant to zero or that it bounds.

The cobordism of Lefschetz fibrations is clearly an equivalence relation. We denote by $\Lambda(g, m)$ the set of equivalence classes. We remark that we are considering only oriented, compact, not necessarily connected Lefschetz fibrations.

There is a general theory of (co)bordism in several flavors. The book of Conner and Floyd [6] is a good reference for general bordism theory. On the other hand, [2] considers cobordisms of maps having only singularities of some prescribed class specified by an invariant open subset of the space of $k$-jets. However, Lefschetz fibrations do not seem to fit well in this general setting, because of the rigidity of Lefschetz fibrations between closed manifolds. In [23], both the source and the target are allowed to change up to cobordism. In these theories there is no control over the fiber. However, to the author's knowledge, there is no similar theory specific to Lefschetz fibrations.

Definition 4.2 The sum of two cobordism classes is defined by

$$
\left[f_{1}\right]+\left[f_{2}\right]=\left[f_{1} \sqcup f_{2}: V_{1} \sqcup V_{2} \rightarrow M_{1} \sqcup M_{2}\right] .
$$

It turns out that this operation is well-defined (does not depend on the representatives), and $\Lambda(g, m)$ with this operation is an abelian group which we call the Lefschetz cobordism group of genus $g$ and dimension $m$. The identity element is the empty fibration (or equivalently, the class of a Lefschetz fibration that bounds), and the inverse is given by $-[f]=[-f]$. Indeed, $f-f$ bounds $f \times \operatorname{id}_{I}: V \times I \rightarrow M \times I$. 
We define another operation on $\Lambda(g, m)$. Let $D_{i} \subset M_{i}-A_{f_{i}}$ be a small ball, for $i=1,2$. So, $f_{i}$ is a trivial bundle over $D_{i}$, that is, $f_{i}^{-1}\left(D_{i}\right)$ can be identified with $D_{i} \times F_{g}$.

Let $M_{1} \# M_{2}=\left(M_{1}-\operatorname{Int}\left(D_{1}\right)\right) \cup_{\partial}\left(M_{2}-\operatorname{Int}\left(D_{2}\right)\right)$ be the result of the identification $D_{1} \cong-D_{2}$ restricted to the boundary, that is the ordinary connected sum. Also let $V_{1} \#_{F_{g}} V_{2}=\left(V_{1}-\right.$ Int $\left.f_{1}^{-1}\left(D_{1}\right)\right) \cup_{\partial}\left(V_{2}-\right.$ Int $\left.f_{2}^{-1}\left(D_{2}\right)\right)$ be the result of the identification $f_{1}^{-1}\left(D_{1}\right) \cong D_{1} \times F_{g} \cong-D_{2} \times F_{g} \cong-f_{2}^{-1}\left(D_{2}\right)$, again restricted to the boundary.

Definition 4.3 The fiber sum of $f_{1}$ and $f_{2}$ is the Lefschetz fibration

$$
f_{1} \# f_{2}: V_{1} \#_{F_{g}} V_{2} \rightarrow M_{1} \# M_{2}
$$

defined by $f_{i}$ on $V_{i}-\operatorname{Int}\left(f_{i}^{-1}\left(D_{i}\right)\right), i=1,2$.

Note that, in general, the fiber sum operation depends on the choice of a gluing diffeomorphism $h \in \mathcal{M}_{g}$ that occurs in the above identification between the preimages of the balls. Actually, there is not a canonical choice for $h$. However, the following holds.

Proposition 4.4 We have $\left[f_{1}\right]+\left[f_{2}\right]=\left[f_{1} \# f_{2}\right]$. Therefore, the fiber sum does not depend on the choice of the attaching diffeomorphism up to cobordism. It follows that any class in $\Lambda(g, m)$ has a connected representative.

Proof Take the product $\left(M_{1} \sqcup M_{2}\right) \times I$, and add an orientable 1-handle $H^{1}=B^{1} \times B^{m}$ to it, with attaching region $\left(D_{1} \sqcup D_{2}\right) \times\{1\}$. Also glue $H^{1} \times F$ to $\left(V_{1} \sqcup V_{2}\right) \times I$ along $\left(f_{1}^{-1}\left(D_{1}\right) \sqcup f_{2}^{-1}\left(D_{2}\right)\right) \times\{1\} \cong\left(D_{1} \sqcup D_{2}\right) \times F_{g}$ with a fibered attaching diffeomorphism such that the fiber is mapped onto itself by the identity on $D_{1} \times F_{g}$ and by the attaching diffeomorphism occurring in the fiber sum on $D_{2} \times F_{g}$. We get a cobordism between $f_{1}+f_{2}$ and $f_{1} \# f_{2}$, and this concludes the proof.

There is an obvious forgetful homomorphism $\Phi: \Lambda(g, m) \rightarrow \Omega_{m+2}^{\mathrm{SO}} \oplus \Omega_{m}^{\mathrm{SO}}$ defined by $\Phi([f: V \rightarrow M])=([V],[M])$. This is surjective on the second component, since for any $M$ there is the trivial fibration $M \times F_{g} \rightarrow M$.

Now we consider the case $m=2$. For $f: V \rightarrow S$ let $n_{+}(f)$ be the number of positive critical points of $f$, and let $n_{-}(f)$ be the number of negative critical points. There are two canonical homomorphisms $\sigma, \eta: \Lambda(g, 2) \rightarrow \mathbb{Z}$, defined by $\sigma([f])=\operatorname{Sign}(V)$, and $\eta([f])=n_{+}(f)-n_{-}(f)$.

Proposition 4.5 $\sigma$ and $\eta$ are well-defined homomorphisms. 
Proof It is obvious that $\sigma$ is well defined and a homomorphism. Let us prove the proposition for $\eta$. Let $h: W \rightarrow Y$ be a cobordism between $f_{1}: V_{1} \rightarrow S_{1}$ and $f_{2}: V_{2} \rightarrow S_{2}$. The critical image of $h$ is a properly embedded compact curve in the 3-manifold $Y$. So, $A_{h}$ is a disjoint union of circles and arcs. Circles do not contribute to $\eta$. If an arc has both endpoints in $S_{1}$ (or in $S_{2}$ ), these are two opposite critical points of $f_{1}$ (or $f_{2}$ ), so they cancel. If there is one endpoint in $S_{1}$ and the other in $S_{2}$, these are critical points of, respectively, $f_{1}$ and $f_{2}$ of the same sign. Since any critical point of $f_{i}$ is the endpoint of an arc, we get $\eta\left(f_{1}\right)=\eta\left(f_{2}\right)$, and so $\eta$ is well defined. That $\eta$ is a homomorphism is immediate.

Remark By results in [11], $\sigma$ and $\eta$ are surjective for $g \geq 2$. In fact, it is proved that any lantern relation contributes \pm 1 to the signature and (obviously) to $\eta$, so by putting sufficiently many lantern relations or its inverses, in the monodromy sequence of a Lefschetz fibration over the sphere, we realize all signatures and get the surjectivity of $\eta$. These fibrations are achiral.

Remark The signature defines an isomorphism $\Omega_{4}^{\mathrm{SO}} \cong \mathbb{Z}$. So, $\sigma$ is equivalent to the forgetful homomorphism $\Phi$.

We conclude by showing a remarkable relation with the singular bordism groups, implying that $\Lambda(g, 2)$ and $\Lambda(g, 3)$ are finitely generated.

Let $\Omega_{n}(X)$ denote the $n$-dimensional singular bordism group of $X$. Recall that the elements of $\Omega_{n}(X)$ are the bordism classes of oriented singular $n$-manifolds in $X$, that is, pairs of the form $(N, q)$, with $N$ a closed oriented $n$-manifold, and $q: N \rightarrow X$. These groups can be expressed in terms of singular homology with coefficients in the cobordism ring $\Omega_{*}^{\mathrm{SO}}$, modulo odd torsion [6].

Proposition 4.6 Let $f: V \rightarrow M$ be a Lefschetz fibration with fiber $F_{g}$. For any $n$ there is a canonical homomorphism

$$
f_{*}: \Omega_{n}(M) \rightarrow \Lambda(g, n), \quad f_{*}([(N, q)])=\left[q^{*}(f)\right] .
$$

Proof Let $(N, q)$ be a representative of a class of $\Omega_{n}(M)$, so $q: N \rightarrow M$. Up to a small homotopy we can assume that $q$ is $f$-regular. Then we can take the pullback $q^{*}(f)$.

If $\left(N^{\prime}, q^{\prime}\right)$ is bordant to $(N, q)$, where $q^{\prime}: N^{\prime} \rightarrow M$ is $f$-regular, there is a bordism $Q: Y \rightarrow M$, where $Y$ is a cobordism between $N$ and $N^{\prime}$, and $Q_{\mid \partial Y}=q \sqcup\left(-q^{\prime}\right)$. Up to homotopy relative to the boundary, we can assume that $Q$ is $f$-regular. Then, $Q^{*}(f)$ is a cobordism between $q^{*}(f)$ and $\left(q^{\prime}\right)^{*}(f)$. 
It follows that the map $f_{*}: \Omega_{n}(M) \rightarrow \Lambda(g, n), f_{*}([(N, q)])=\left[q^{*}(f)\right]$, is a welldefined homomorphism.

Corollary 4.7 If $u: U \rightarrow M$ is universal with respect to Lefschetz fibrations over $n$-manifolds, then $u_{*}: \Omega_{n}(M) \rightarrow \Lambda(g, n)$ is surjective. Therefore, there are epimorphisms $u_{n *}: \Omega_{n}\left(M_{n}\right) \rightarrow \Lambda(g, n)$ for $n=2,3$, with $u_{n}: U_{n} \rightarrow M_{n}$ the two universal Lefschetz fibrations constructed in Section 3.

Proof Let $[f: V \rightarrow N], \operatorname{dim} N=n$, be an element of $\Lambda(g, n)$. Since $u$ is universal, there is $q: N \rightarrow M$ such that $f=q^{*}(u)$, and so $[f]=u_{*}([(N, q)])$.

Note that there is also the epimorphism $u_{3 *}: \Omega_{2}\left(M_{3}\right) \rightarrow \Lambda(g, 2)$.

Corollary 4.8 There is an epimorphism $\lambda: H_{2}\left(M_{2}\right) \rightarrow \Lambda(g, 2)$.

Proof The canonical homomorphism

$$
\mu: \Omega_{2}\left(M_{2}\right) \rightarrow H_{2}\left(M_{2}\right), \quad \mu([(N, q)])=q_{*}([N])
$$

is an isomorphism because $H_{*}\left(M_{2}\right)$ has no torsion and $H_{i}\left(M_{2}\right)=0$ for $i \neq 0,2$ (indeed, $M_{2}$ is $B^{4}$ union 2-handles) [6, Chapter II]. Therefore, $u_{2 *} \circ \mu^{-1}$ is an epimorphism.

\section{References}

[1] S Akbulut, B Ozbagci, Lefschetz fibrations on compact Stein surfaces, Geom. Topol. 5 (2001) 319-334 MR1825664

[2] Y Ando, Cobordisms of maps with singularities of given class, Algebr. Geom. Topol. 8 (2008) 1989-2029 MR2449006

[3] N Apostolakis, R Piergallini, D Zuddas, Lefschetz fibrations over the disc, Proc. Lond. Math. Soc. 107 (2013) 340-390 MR3092341

[4] R İ Baykur, Existence of broken Lefschetz fibrations, Int. Math. Res. Not. 2008 (2008) 15 MR2439543

[5] R İ Baykur, Topology of broken Lefschetz fibrations and near-symplectic fourmanifolds, Pacific J. Math. 240 (2009) 201-230 MR2485463

[6] P E Conner, E E Floyd, Differentiable periodic maps, Ergeb. Math. Grenzgeb. 33, Springer, Berlin (1964) MR0176478

[7] A J Di Scala, N Kasuya, D Zuddas, On embeddings of almost complex manifolds in almost complex Euclidean spaces arXiv:1410.1321 
[8] S K Donaldson, Lefschetz pencils on symplectic manifolds, J. Differential Geom. 53 (1999) 205-236 MR1802722

[9] C J Earle, J Eells, The diffeomorphism group of a compact Riemann surface, Bull. Amer. Math. Soc. 73 (1967) 557-559 MR0212840

[10] C J Earle, J Eells, A fibre bundle description of Teichmüller theory, J. Differential Geometry 3 (1969) 19-43 MR0276999

[11] H Endo, S Nagami, Signature of relations in mapping class groups and nonholomorphic Lefschetz fibrations, Trans. Amer. Math. Soc. 357 (2005) 3179-3199 MR2135741

[12] B Farb, D Margalit, A primer on mapping class groups, Princeton Math. Series 49, Princeton Univ. Press (2012) MR2850125

[13] T Fuller, Hyperelliptic Lefschetz fibrations and branched covering spaces, Pacific J. Math. 196 (2000) 369-393 MR1800582

[14] D T Gay, R Kirby, Indefinite Morse 2-functions; broken fibrations and generalizations arXiv: 1102.0750

[15] D T Gay, R Kirby, Constructing Lefschetz-type fibrations on four-manifolds, Geom. Topol. 11 (2007) 2075-2115 MR2350472

[16] D T Gay, R Kirby, Reconstructing 4-manifolds from Morse 2-functions, from: "Proceedings of the Freedman Fest”, (R Kirby, V Krushkal, Z Wang, editors), Geom. Topol. Monogr. 18 (2012) 103-114 MR2350472

[17] R E Gompf, A I Stipsicz, 4-manifolds and Kirby calculus, Graduate Studies in Mathematics 20, Amer. Math. Soc. (1999) MR1707327

[18] A Gramain, Le type d'homotopie du groupe des difféomorphismes d'une surface compacte, Ann. Sci. École Norm. Sup. 6 (1973) 53-66 MR0326773

[19] S Kamada, Y Matsumoto, T Matumoto, K Waki, Chart description and a new proof of the classification theorem of genus one Lefschetz fibrations, J. Math. Soc. Japan 57 (2005) 537-555 MR2123244

[20] A Loi, R Piergallini, Compact Stein surfaces with boundary as branched covers of $B^{4}$, Invent. Math. 143 (2001) 325-348 MR1835390

[21] Y Matsumoto, On 4-manifolds fibered by tori, Proc. Japan Acad. Ser. A Math. Sci. 58 (1982) 298-301 MR682687

[22] B Moishezon, Complex surfaces and connected sums of complex projective planes, Lecture Notes in Mathematics 603, Springer, Berlin (1977) MR0491730

[23] R E Stong, Cobordism of maps, Topology 5 (1966) 245-258 MR0196759

[24] D Zuddas, Representing Dehn twists with branched coverings, Int. Math. Res. Not. 2009 (2009) 387-413 MR2482119 
[25] D Zuddas, Universal Lefschetz fibrations over bounded surfaces, Algebr. Geom. Topol. 12 (2012) 1811-1829 MR2979999

School of Mathematics, Korea Institute for Advanced Study 85 Hoegiro, Dongdaemun-gu 02455, South Korea

d.zuddas@gmail.com, zuddas@kias.re.kr http://newton.kias.re.kr/ zuddas

Received: 16 November 2014 